MATHEMATICS OF COMPUTATION

Volume 77, Number 262, April 2008, Pages 985-994

S 0025-5718(07)02032-7

Article electronically published on November 14, 2007

\title{
MAXIMUM OF THE MODULUS OF KERNELS IN GAUSS-TURÁN QUADRATURES
}

\author{
GRADIMIR V. MILOVANOVIĆ, MIODRAG M. SPALEVIĆ, AND MIROSLAV S. PRANIĆ
}

\begin{abstract}
We study the kernels $K_{n, s}(z)$ in the remainder terms $R_{n, s}(f)$ of the Gauss-Turán quadrature formulae for analytic functions on elliptical contours with foci at \pm 1 , when the weight $\omega$ is a generalized Chebyshev weight function. For the generalized Chebyshev weight of the first (third) kind, it is shown that the modulus of the kernel $\left|K_{n, s}(z)\right|$ attains its maximum on the real axis (positive real semi-axis) for each $n \geq n_{0}, n_{0}=n_{0}(\rho, s)$. It was stated as a conjecture in [Math. Comp. 72 (2003), 1855-1872]. For the generalized Chebyshev weight of the second kind, in the case when the number of the nodes $n$ in the corresponding Gauss-Turán quadrature formula is even, it is shown that the modulus of the kernel attains its maximum on the imaginary axis for each $n \geq n_{0}, n_{0}=n_{0}(\rho, s)$. Numerical examples are included.
\end{abstract}

\section{INTRODUCTION}

We consider the Gauss-Turán quadrature formula with multiple nodes

$$
\int_{-1}^{1} f(t) \omega(t) d t=\sum_{\nu=1}^{n} \sum_{i=0}^{2 s} A_{i, \nu} f^{(i)}\left(\tau_{\nu}\right)+R_{n, s}(f) \quad\left(n \in \mathbb{N} ; s \in \mathbb{N}_{0}\right)
$$

where $\omega$ is a nonnegative and integrable function on the interval $(-1,1)$, which is exact for all algebraic polynomials of degree at most $2(s+1) n-1$. The nodes $\tau_{\nu}$ in (1.1) must be zeros of the $s$-orthogonal polynomials with respect to the weight function $\omega(t)$. The $s$-orthogonal polynomials $\pi_{n}=\pi_{n, s}$ with respect to the weight function $\omega(t)$ are polynomials which satisfy the following orthogonality conditions:

$$
\int_{-1}^{1} \pi_{n}(t)^{2 s+1} t^{k} \omega(t) d t=0, \quad k=0,1, \ldots, n-1 .
$$

Numerically stable methods for constructing nodes $\tau_{\nu}$ and coefficients $A_{i, \nu}$ can be found in [3, 10, 13. For more details on quadrature formulae with multiple nodes see [7] and [9].

Received by the editor August 15, 2006 and, in revised form, December 4, 2006.

1991 Mathematics Subject Classification. Primary 41A55; Secondary 65D30, 65D32.

Key words and phrases. Gauss-Turán quadrature, Chebyshev weight functions, remainder term for analytic functions, error estimate, contour integral representation, confocal ellipses, kernel.

The authors were supported in part by the Swiss National Science Foundation (SCOPES Joint Research Project No. IB7320-111079 "New Methods for Quadrature") and the Serbian Ministry of Science (Research Projects: "Approximation of linear operators" (No. \#144005) \& "Orthogonal systems and applications" (No. \#144004C)). 
Let $\Gamma$ be a simple closed curve in the complex plane surrounding the interval $[-1,1]$ and let $D$ be its interior. If the integrand $f$ is analytic on $D$ and continuous on $\bar{D}$, then the remainder term $R_{n, s}$ in (1.1) admits the contour integral representation (see [14, [11])

$$
R_{n, s}(f)=\frac{1}{2 \pi i} \oint_{\Gamma} K_{n, s}(z) f(z) d z .
$$

The kernel is given by $K_{n, s}(z)=\rho_{n, s}(z) /\left[\pi_{n, s}(z)\right]^{2 s+1}, z \notin[-1,1]$, where

$$
\rho_{n, s}(z)=\int_{-1}^{1} \frac{\left[\pi_{n, s}(t)\right]^{2 s+1}}{z-t} \omega(t) d t .
$$

The modulus of the kernel is symmetric with respect to the real axis, i.e., $\left|K_{n, s}(\bar{z})\right|=\left|K_{n, s}(z)\right|$. If the weight function in (1.1) is even, the modulus of the kernel is symmetric with respect to both axes, i.e., $\left|K_{n, s}(-\bar{z})\right|=\left|K_{n, s}(z)\right|$ (see [11, Lemma 2.1]).

A particularly interesting case is the Chebyshev weight $\omega_{1}(t)=\left(1-t^{2}\right)^{-1 / 2}$. In 1930, S. Bernstein [1] showed that the monic Chebyshev polynomial $\hat{T}_{n}(t)=$ $T_{n}(t) / 2^{n-1}$ minimizes all integrals of the form

$$
\int_{-1}^{1} \frac{\left|\pi_{n}(t)\right|^{k+1}}{\sqrt{1-t^{2}}} d t \quad(k \geq 0) .
$$

This means that the Chebyshev polynomials $T_{n}$ are $s$-orthogonal on $(-1,1)$ for each $s \geq 0$. Ossicini and Rosati [14] found three other weights $\omega_{k}(t)(k=2,3,4)$ for which the $s$-orthogonal polynomials can be identified as Chebyshev polynomials of the second, third and fourth kind: $U_{n}, V_{n}$, and $W_{n}$, which are defined by

$$
U_{n}(\cos \theta)=\frac{\sin (n+1) \theta}{\sin \theta}, \quad V_{n}(\cos \theta)=\frac{\cos \left(n+\frac{1}{2}\right) \theta}{\cos \frac{1}{2} \theta}, \quad W_{n}(\cos \theta)=\frac{\sin \left(n+\frac{1}{2}\right) \theta}{\sin \frac{1}{2} \theta},
$$

respectively (cf. Gautschi and Notaris [4]). However, these weights depend on $s$,

$$
\omega_{2}(t)=\left(1-t^{2}\right)^{1 / 2+s}, \quad \omega_{3}(t)=\frac{(1+t)^{1 / 2+s}}{(1-t)^{1 / 2}}, \quad \omega_{4}(t)=\frac{(1-t)^{1 / 2+s}}{(1+t)^{1 / 2}} .
$$

It is easy to see that $W_{n}(-t)=(-1)^{n} V_{n}(t)$, so that in the investigation it is sufficient to study only the first three generalized Chebyshev weights $\omega_{k}(t), k=1,2,3$.

The integral representation (1.2) leads directly to the error estimate

$$
\left|R_{n, s}\right| \leq \frac{l(\Gamma)}{2 \pi}\left(\max _{z \in \Gamma}\left|K_{n, s}(z)\right|\right)\left(\max _{z \in \Gamma}|f(z)|\right),
$$

where $l(\Gamma)$ denotes the length of the contour $\Gamma$. First maximum depends only on the quadrature rule (i.e., on $\omega$ ) and not on $f$. The first unified approach described above was taken by Donaldson and Elliot [2]. They applied it to several kinds of interpolatory and non-interpolatory quadrature rules. Error bounds for Gaussian quadratures of analytic functions were studied by Gautschi and Varga [5] (see also [6]), and later by Schira [15, 16, Hunter and Nikolov [8].

As a contour $\Gamma$ we take an ellipse $\mathcal{E}_{\rho}$ with foci at points \pm 1 and a sum of semi-axes $\rho>1$

$$
\mathcal{E}_{\rho}=\left\{z \in \mathbb{C}: \quad z=\frac{1}{2}\left(u+u^{-1}\right), \quad 0 \leq \theta \leq 2 \pi\right\}, \quad u=\rho e^{i \theta}
$$


When $\rho \rightarrow 1$, the ellipse shrinks to the interval $[-1,1]$, while with increasing $\rho$ it becomes more and more circle-like.

When $\omega$ is the generalized Chebyshev weight of the first (third) kind, it is conjectured, on the basis of numerical experiments (see [11]), that the modulus of the kernel attains its maximum on the real axis (positive real semi-axis) for each $n \geq n_{0}, n_{0}=n_{0}(\rho, s)$.

In this paper we prove those conjectures. Moreover, for the generalized Chebyshev weight of the second kind, in the case when the number of the nodes $n$ in the corresponding Gauss-Turán quadrature formula is even, we show that the modulus of the kernel attains its maximum on the imaginary axis for each $n \geq n_{0}, n_{0}=$ $n_{0}(\rho, s)$. Numerical examples are included.

\section{The MaXimum MOdulus of THE KeRnel on CONFOCAL ELLIPSES}

We study the magnitude of $\left|K_{n, s}(z)\right|$ on the contour $\mathcal{E}_{\rho}$ for the generalized Chebyshev weight functions of the first, second and third kind, respectively. The particular case $\left|K_{n, 0}(z)\right|$ was analyzed in details by Gautschi et al. [5, 6].

2.1. The weight function $\omega_{1}(t)=\left(1-t^{2}\right)^{-1 / 2}$. An explicit representation of the kernel $K_{n, s}^{(1)}(z)$ on the ellipse $\mathcal{E}_{\rho}$ for the weight function $\omega_{1}(t)$ was given by Milovanović and Spalević in [11, as well as

$$
\left|K_{n, s}^{(1)}(z)\right|=\frac{2^{1-s} \pi}{\rho^{n}} \frac{\left|Z_{n, s}^{(1)}\left(\rho e^{i \theta}\right)\right|}{\left(a_{2}-\cos 2 \theta\right)^{1 / 2}\left(a_{2 n}+\cos 2 n \theta\right)^{1 / 2+s}}, \quad z \in \mathcal{E}_{\rho},
$$

where

$$
a_{j}=a_{j}(\rho)=\frac{1}{2}\left(\rho^{j}+\rho^{-j}\right), \quad j \in \mathbb{N}
$$

and

$$
Z_{n, s}^{(1)}(u)=\sum_{k=0}^{s}\left(\begin{array}{c}
2 s+1 \\
s+k+1
\end{array}\right) u^{-2 n k}=\sum_{k=0}^{s}\left(\begin{array}{c}
2 s+1 \\
k
\end{array}\right) u^{-2 n(s-k)} .
$$

The weight function $\omega_{1}(t)$ is even, so we can take $\theta \in[0, \pi / 2]$.

The following result was conjectured in [11]:

Theorem 2.1. For each fixed $\rho>1$ and $s \in \mathbb{N}_{0}$ there exists $n_{0}=n_{0}(\rho, s)$ such that

$$
\max _{z \in \mathcal{E}_{\rho}}\left|K_{n, s}^{(1)}(z)\right|=K_{n, s}^{(1)}\left(\frac{1}{2}\left(\rho+\rho^{-1}\right)\right)
$$

for each $n \geq n_{0}$.

Proof. The inequality $\left|Z_{n, s}^{(1)}\left(\rho e^{i \theta}\right)\right| \leq Z_{n, s}^{(1)}(\rho)$ immediately follows from (2.3). Because of that and (2.1), it is sufficient to prove

$$
\frac{1}{\left(a_{2}-\cos 2 \theta\right)^{1 / 2}\left(a_{2 n}+\cos 2 n \theta\right)^{1 / 2+s}} \leq \frac{1}{\left(a_{2}-1\right)^{1 / 2}\left(a_{2 n}+1\right)^{1 / 2+s}}
$$

for a sufficiently large $n\left(n \geq n_{0}(\rho, s)\right)$ and $\theta \in(0, \pi / 2]$, where $a_{j}$ are given by (2.2) .

By squaring (2.4) it is reduced to

$$
\left(a_{2}-1\right)\left(a_{2 n}+1\right)^{2 s+1} \leq\left(a_{2}-\cos 2 \theta\right)\left(a_{2 n}+\cos 2 n \theta\right)^{2 s+1} .
$$

The following transformation will be used

$$
a_{2}-\cos 2 \theta=\left(a_{2}-1\right)+2 \sin ^{2} \theta .
$$


Further, we will use

$$
\begin{aligned}
\left(a_{2 n}+\cos 2 n \theta\right)^{2 s+1} & =\left(\left(a_{2 n}+1\right)-2 \sin ^{2} n \theta\right)^{2 s+1} \\
& =\left(a_{2 n}+1\right)^{2 s+1}+\sum_{k=1}^{2 s+1}(-2)^{k}\left(\begin{array}{c}
2 s+1 \\
k
\end{array}\right)\left(a_{2 n}+1\right)^{2 s+1-k} \sin ^{2 k} n \theta,
\end{aligned}
$$

i.e.,

$$
\left(a_{2 n}+\cos 2 n \theta\right)^{2 s+1}=\left(a_{2 n}+1\right)^{2 s+1}-2\left(\sin ^{2} n \theta\right) E_{\rho, s}(n, \theta),
$$

where

$$
E_{\rho, s}(n, \theta)=\sum_{k=1}^{2 s+1}(-2)^{k-1}\left(\begin{array}{c}
2 s+1 \\
k
\end{array}\right)\left(a_{2 n}+1\right)^{2 s+1-k} \sin ^{2 k-2} n \theta \quad(\geq 0) .
$$

It is easy to see that $E_{\rho, s}(n, \theta)$ can be represented in the form

$$
E_{\rho, s}(n, \theta)=(2 s+1)\left(a_{2 n}+1\right)^{2 s}+\sum_{k=2}^{2 s+1}(-2)^{k-1}\left(\begin{array}{c}
2 s+1 \\
k
\end{array}\right)\left(a_{2 n}+1\right)^{2 s+1-k} \sin ^{2 k-2} n \theta,
$$

i.e.,

$$
\begin{aligned}
E_{\rho, s}(n, \theta)=(2 s+1)\left(a_{2 n}+1\right)^{2 s} & \\
& -\sum_{k=1}^{s} 2^{2 k-1}\left(\begin{array}{c}
2 s+1 \\
2 k
\end{array}\right)\left(a_{2 n}+1\right)^{2 s-2 k+1} \sin ^{4 k-2} n \theta \\
& +\sum_{k=1}^{s} 2^{2 k}\left(\begin{array}{c}
2 s+1 \\
2 k+1
\end{array}\right)\left(a_{2 n}+1\right)^{2 s-2 k} \sin ^{4 k} n \theta .
\end{aligned}
$$

Using (2.6) and (2.7), the inequality (2.5) is reduced to

$$
\begin{aligned}
& \left(a_{2}-1\right)\left(a_{2 n}+1\right)^{2 s+1} \\
& \quad \leq\left[\left(a_{2}-1\right)+2 \sin ^{2} \theta\right]\left[\left(a_{2 n}+1\right)^{2 s+1}-2\left(\sin ^{2} n \theta\right) E_{\rho, s}(n, \theta)\right],
\end{aligned}
$$

i.e.,

$$
2 \sin ^{2} \theta\left(a_{2 n}+1\right)^{2 s+1}-2 \sin ^{2} n \theta\left[\left(a_{2}-1\right)+2 \sin ^{2} \theta\right] E_{\rho, s}(n, \theta) \geq 0 .
$$

Dividing this inequality by $2 \sin ^{2} \theta$, it becomes

$$
\left(a_{2 n}+1\right)^{2 s+1}-\frac{\sin ^{2} n \theta}{\sin ^{2} \theta}\left[\left(a_{2}-1\right)+2 \sin ^{2} \theta\right] E_{\rho, s}(n, \theta) \geq 0 .
$$

By using the well-known fact $|\sin n \theta / \sin \theta| \leq n$, it is easy to see that

$$
\frac{\sin ^{2} n \theta}{\sin ^{2} \theta}\left[\left(a_{2}-1\right)+2 \sin ^{2} \theta\right]=\left(a_{2}-1\right) \frac{\sin ^{2} n \theta}{\sin ^{2} \theta}+2 \sin ^{2} n \theta \leq\left(a_{2}-1\right) n^{2}+2 .
$$

According to (2.8), we conclude that

$$
\begin{aligned}
E_{\rho, s}(n, \theta)-(2 s+1)\left(a_{2 n}+1\right)^{2 s}= & \sum_{k=1}^{s} \frac{4^{k}(2 s+1) !}{(2 k) !(2 s-2 k) !}\left(a_{2 n}+1\right)^{2 s-2 k} \\
& \times\left(\frac{\sin ^{2} n \theta}{2 k+1}-\frac{a_{2 n}+1}{2(2 s-2 k+1)}\right) \sin ^{4 k-2} n \theta .
\end{aligned}
$$

Since $\sin ^{4 k-2} n \theta \leq 1$ and

$$
\frac{\sin ^{2} n \theta}{2 k+1}-\frac{a_{2 n}+1}{2(2 s-2 k+1)} \leq \frac{1}{2 k+1}-\frac{a_{2 n}+1}{2(2 s-2 k+1)},
$$


from the previous equality we obtain

$$
E_{\rho, s}(n, \theta)-(2 s+1)\left(a_{2 n}+1\right)^{2 s} \leq \sum_{k=1}^{s} 4^{k}\left(a_{2 n}+1\right)^{2 s-2 k}\left[\left(\begin{array}{c}
2 s+1 \\
2 k+1
\end{array}\right)-\frac{a_{2 n}+1}{2}\left(\begin{array}{c}
2 s+1 \\
2 k
\end{array}\right)\right] .
$$

Therefore,

$$
E_{\rho, s}(n, \theta) \leq \sum_{k=0}^{s} 4^{k}\left(\begin{array}{l}
2 s+1 \\
2 k+1
\end{array}\right)\left(a_{2 n}+1\right)^{2 s-2 k}-\frac{1}{2} \sum_{k=1}^{s} 4^{k}\left(\begin{array}{c}
2 s+1 \\
2 k
\end{array}\right)\left(a_{2 n}+1\right)^{2 s-2 k+1} .
$$

Using the last inequality and (2.10), we conclude that the left-hand side of (2.9) is greater than or equal to $F(n) \equiv F_{\rho, s}(n)$, where

$$
\begin{aligned}
F_{\rho, s}(n) & :=\left(a_{2 n}+1\right)^{2 s+1}-\left[\left(a_{2}-1\right) n^{2}+2\right] \\
\times & {\left[\sum_{k=0}^{s} 4^{k}\left(\begin{array}{c}
2 s+1 \\
2 k+1
\end{array}\right)\left(a_{2 n}+1\right)^{2 s-2 k}-\frac{1}{2} \sum_{k=1}^{s} 4^{k}\left(\begin{array}{c}
2 s+1 \\
2 k
\end{array}\right)\left(a_{2 n}+1\right)^{2 s-2 k+1}\right] . }
\end{aligned}
$$

Since $F_{\rho, s}(n)\left(\rho, s\right.$ - are fixed) is continuous on $\mathbb{R}$ and $\lim _{n \rightarrow+\infty} F_{\rho, s}(n)=+\infty$, it follows that $F_{\rho, s}(n)>0$, for each $n>t$, where $t$ is the largest zero of $F_{\rho, s}(n)$. For $n_{0}$ we can take $[t]+1$.

TABLE 1. The smallest possible (s.p.) values of $n_{0}$ and their approximations $[t]+1(t$ is the largest zero of $F)$

\begin{tabular}{c|cc|cc}
\multirow{2}{*}{$s$} & \multicolumn{2}{|c|}{$\rho=1.05$} & \multicolumn{2}{c}{$\rho=1.3$} \\
\cline { 2 - 5 } & {$[t]+1$} & the s.p. $n_{0}$ & {$[t]+1$} & the s.p. $n_{0}$ \\
\hline 1 & 41 & 34 & 8 & 7 \\
2 & 50 & 46 & 10 & 9 \\
3 & 56 & 53 & 11 & 10 \\
4 & 59 & 57 & 12 & 11 \\
5 & 63 & 61 & 12 & 12 \\
6 & 65 & 63 & 13 & 12 \\
7 & 67 & 66 & 13 & 13 \\
8 & 69 & 68 & 13 & 13 \\
9 & 70 & 69 & 14 & 13
\end{tabular}

The proof of Theorem 2.1 is not only of theoretical, but also of practical importance. We can use the function $F(n)$ from the proof to estimate $n_{0}$. Numerical values of $[t]+1$ ( $t$ is the largest zero of $F$ ) for some values of $\rho$ and $s$ are presented in Tables 1 and 2, The smallest possible (s.p.) values of $n_{0}$ are also presented. We can see that the smallest possible $n_{0}$ is estimated by $[t]+1$ very well.

A typical graph illustrating the relationship between $n$ and $F(n)$ is given in Figure 1. Here, $\rho=1.05, s=1 ; n \in[1,42]$.

2.2. The weight function $\omega_{2}(t)=\left(1-t^{2}\right)^{s+1 / 2}, s \in \mathbb{N}_{0}$. An explicit representation of the kernel $K_{n, s}^{(2)}(z)$ on the ellipse $\mathcal{E}_{\rho}$ for the weight function $\omega_{2}(t)$ was given in [11], as well as

$$
\left|K_{n, s}^{(2)}(z)\right|=\frac{\pi}{4^{s} \rho^{n+1}}\left[\frac{a_{2}-\cos 2 \theta}{a_{2 n+2}-\cos (2 n+2) \theta}\right]^{s+1 / 2}\left|Z_{n, s}^{(2)}\left(\rho e^{i \theta}\right)\right|,
$$


TABLE 2. The smallest possible (s.p.) values of $n_{0}$ and their approximations $[t]+1(t$ is the largest zero of $F)$

\begin{tabular}{c|cc|cc}
\multirow{2}{*}{$\rho$} & \multicolumn{2}{|c|}{$s=1$} & \multicolumn{2}{|c}{$s=5$} \\
\cline { 2 - 5 } & {$[t]+1$} & the s.p. $n_{0}$ & {$[t]+1$} & the s.p. $n_{0}$ \\
\hline 1.01 & 200 & 165 & 305 & 295 \\
1.02 & 101 & 83 & 153 & 148 \\
1.03 & 68 & 56 & 103 & 100 \\
1.04 & 51 & 42 & 78 & 75 \\
1.05 & 41 & 34 & 63 & 61 \\
1.06 & 35 & 29 & 52 & 51 \\
1.07 & 30 & 25 & 45 & 44 \\
1.08 & 26 & 22 & 40 & 39 \\
1.09 & 24 & 20 & 36 & 35 \\
1.1 & 21 & 18 & 32 & 31 \\
1.2 & 11 & 10 & 17 & 17 \\
1.3 & 8 & 7 & 12 & 12 \\
1.4 & 6 & 6 & 10 & 9 \\
1.5 & 6 & 5 & 8 & 8 \\
1.6 & 5 & 4 & 7 & 7 \\
1.7 & 4 & 4 & 6 & 6 \\
1.8 & 4 & 4 & 6 & 6 \\
1.9 & 4 & 3 & 5 & 5 \\
2.0 & 4 & 3 & 5 & 5
\end{tabular}

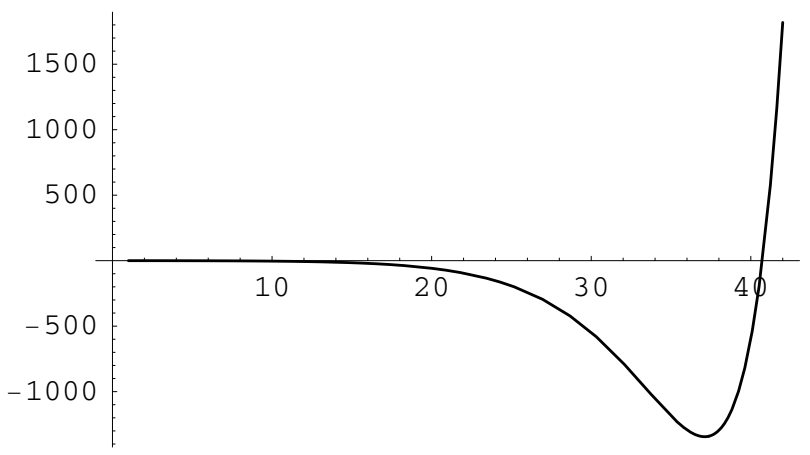

Figure 1. The typical graph of $F(n)$.

where

$$
Z_{n, s}^{(2)}\left(\rho e^{i \theta}\right)=\sum_{k=0}^{s}(-1)^{k}\left(\begin{array}{c}
2 s+1 \\
s+k+1
\end{array}\right)\left(\rho e^{i \theta}\right)^{-2(n+1) k} .
$$

There we proved the following statement:

Theorem 2.2. If $\omega_{2}(t)=\left(1-t^{2}\right)^{s+1 / 2}$ on $(-1,1), s \in \mathbb{N}_{0}$, and $n$ is odd, then

$$
\max _{z \in \mathcal{E}_{\rho}}\left|K_{n, s}^{(2)}(z)\right|=\left|K_{n, s}^{(2)}\left(\frac{i}{2}\left(\rho-\rho^{-1}\right)\right)\right| \text {. }
$$

In this section we consider the case when $n$ is even. 
Theorem 2.3. For each fixed $\rho>1$ and $s \in \mathbb{N}_{0}$ there exists even $n_{0}=n_{0}(\rho, s)$ such that

$$
\max _{z \in \varepsilon_{\rho}}\left|K_{n, s}^{(2)}(z)\right|=\left|K_{n, s}^{(2)}\left(\frac{i}{2}\left(\rho-\rho^{-1}\right)\right)\right|
$$

for each even $n \geq n_{0}$.

Proof. First we prove the inequality

$$
\left|Z_{n, s}^{(2)}\left(\rho e^{i \theta}\right)\right| \leq Z_{n, s}^{(2)}(i \rho), \quad \theta \in[0, \pi / 2), n \text { is even. }
$$

We note that (see [11, Eq. (3.13)])

$$
\begin{aligned}
Z_{n, s}^{(2)}(u) & =\sum_{\nu=0}^{[(s-1) / 2]}\left(\sum_{k=2 \nu}^{2 \nu+1}(-1)^{k}\left(\begin{array}{c}
2 s+1 \\
s+k+1
\end{array}\right) u^{-2(n+1) k}\right)+\zeta_{n, s}(u) \\
& =\sum_{\nu=0}^{[(s-1) / 2]}\left(\begin{array}{c}
2 s+1 \\
s+2 \nu+1
\end{array}\right) u^{-4 \nu(n+1)}\left(1-\alpha u^{-2(n+1)}\right)+\zeta_{n, s}(u),
\end{aligned}
$$

where $u=\rho e^{i \theta}, \alpha=(s-2 \nu) /(s+2 \nu+2), 0<\alpha<1$, and

$$
\zeta_{n, s}(u)=\zeta_{n, s}\left(\rho e^{i \theta}\right):= \begin{cases}0 & \text { if } s \text { is odd }, \\ \left(\rho e^{i \theta}\right)^{-2(n+1) s} & \text { if } s \text { is even, }\end{cases}
$$

as well as $\left|\zeta_{n, s}\left(\rho e^{i \theta}\right)\right|=\zeta_{n, s}(i \rho)$.

Since

$$
\left|Z_{n, s}^{(2)}(u)\right| \leq \sum_{\nu=0}^{[(s-1) / 2]}\left(\begin{array}{c}
2 s+1 \\
s+2 \nu+1
\end{array}\right)\left|u^{-4 \nu(n+1)}\left(1-\alpha u^{-2(n+1)}\right)\right|+\left|\zeta_{n, s}(u)\right|,
$$

introducing $q=\alpha \rho^{-2(n+1)}$, now we get

$$
\begin{aligned}
\left|Z_{n, s}^{(2)}(u)\right| & \leq \sum_{\nu=0}^{[(s-1) / 2]}\left(\begin{array}{c}
2 s+1 \\
s+2 \nu+1
\end{array}\right) \rho^{-4 \nu(n+1)} \sqrt{1-2 q \cos (2 n+2) \theta+q^{2}}+\zeta_{n, s}(i \rho) \\
& \leq \sum_{\nu=0}^{[(s-1) / 2]}\left(\begin{array}{c}
2 s+1 \\
s+2 \nu+1
\end{array}\right) \rho^{-4 \nu(n+1)}(1+q)+\zeta_{n, s}(i \rho) \\
& =\sum_{\nu=0}^{[(s-1) / 2]}\left(\begin{array}{c}
2 s+1 \\
s+2 \nu+1
\end{array}\right)(i \rho)^{-4 \nu(n+1)}\left(1-\alpha(i \rho)^{-2(n+1)}\right)+\zeta_{n, s}(i \rho) \\
& =Z_{n, s}^{(2)}(i \rho) .
\end{aligned}
$$

Therefore, in order to prove the statement, on the basis of (2.11) and (2.13), it is sufficient to prove

$$
\frac{a_{2}-\cos 2 \theta}{a_{2 n+2}-\cos (2 n+2) \theta} \leq \frac{a_{2}+1}{a_{2 n+2}+1}, \quad \theta \in[0, \pi / 2), n \text { is even, }
$$

for sufficiently large $n\left(n \geq n_{0} ; n_{0}=n_{0}(\rho)-\right.$ even $)$. This is equivalent to

$$
a_{2 n+2}+a_{2 n+2} \cos 2 \theta-a_{2}-a_{2} \cos (2 n+2) \theta+\cos 2 \theta-\cos (2 n+2) \theta \geq 0,
$$

and furthermore to

$$
a_{2 n+2}(1+\cos 2 \theta)-a_{2}(1+\cos 2(n+1) \theta)+(1+\cos 2 \theta)-(1+\cos 2(n+1) \theta) \geq 0,
$$


introducing half-angles, to $\left(a_{2 n+2}+1\right) \cos ^{2} \theta-\left(a_{2}+1\right) \cos ^{2}(n+1) \theta \geq 0$, and to

$$
\left(a_{2 n+2}+1\right)-\frac{\cos ^{2}(n+1) \theta}{\cos ^{2} \theta}\left(a_{2}+1\right) \geq 0 .
$$

Since $|\cos (n+1) \theta / \cos \theta| \leq n+1$ for even $n$, we have

$$
\left(a_{2 n+2}+1\right)-\frac{\cos ^{2}(n+1) \theta}{\cos ^{2} \theta}\left(a_{2}+1\right) \geq\left(a_{2 n+2}+1\right)-(n+1)^{2}\left(a_{2}+1\right),
$$

which means that (2.14) holds if $\left(a_{2 n+2}+1\right)-(n+1)^{2}\left(a_{2}+1\right) \geq 0$.

Since $a_{2}+1=2 a_{1}^{2}$ and $a_{2 n+2}+1=2 a_{n+1}^{2}$, the last inequality is equivalent to $a_{n+1}^{2}-\left[(n+1) a_{1}\right]^{2} \geq 0$ or to $a_{n+1}-(n+1) a_{1} \geq 0$. Substituting $a_{1}, a_{n+1}$ by (2.2), this inequality becomes

$$
G_{\rho}(n) \equiv G(n):=\rho^{n+1}-(n+1) \rho-(n+1) \rho^{-1}+\rho^{-(n+1)} \geq 0 .
$$

Since $G_{\rho}(n)$ ( $\rho$ - is fixed) is continuous on $\mathbb{R}$ and $\lim _{n \rightarrow+\infty} G_{\rho}(n)=+\infty$, it follows that $G_{\rho}(n)>0$, for each $n>t$, where $t$ is the largest zero of $G_{\rho}(n)$. For $n_{0}$ we can take the smallest even integer which is greater than or equal to $t$.

Let $\bar{t}$ be the smallest even integer $\geq t$. If $t$ is an even integer, we have $\bar{t}=t$, otherwise

$$
\bar{t}:= \begin{cases}{[t]+1} & \text { if }[t] \text { is odd } \\ {[t]+2} & \text { if }[t] \text { is even. }\end{cases}
$$

We can use the function $G(n)$ from the proof to estimate $n_{0}$. Numerical values of $\bar{t}$ for some values of $\rho$ are presented in Table 3 The smallest possible (s.p.) values of $n_{0}$, for $s=1, \ldots, 10$, are also presented in the same table. We can see that the smallest possible $n_{0}$ (which is even) is estimated very well, independently of $s$.

Finally, observe that the function $G_{\rho}(n) \equiv G(n)$ in this case has rather simple form. Because of $G(0)=0$, and $G^{\prime \prime}(n)=2 a_{n+1} \log ^{2} \rho>0$, for $n \in[0,+\infty)$, we conclude that $G(n)$ has at most one zero $t$ in the interval $(0,+\infty)$.

2.3. The weight function $\omega_{3}(t)=(1+t)^{1 / 2+s}(1-t)^{-1 / 2}, s \in \mathbb{N}_{0}$. An explicit representation of the kernel $K_{n, s}^{(3)}(z)$ on the ellipse $\mathcal{E}_{\rho}$ for the generalized Chebyshev weight function of the third kind $\omega_{3}(t)$ was given in [11, as well as

$$
\left|K_{n, s}^{(3)}(z)\right|=\frac{2^{1-s} \pi}{\rho^{n+1 / 2}} \frac{\left(a_{1}+\cos \theta\right)^{s+1}\left|Z_{n, s}^{(3)}\left(\rho e^{i \theta}\right)\right|}{\left(a_{2}-\cos 2 \theta\right)^{1 / 2}\left(a_{2 n+1}+\cos (2 n+1) \theta\right)^{1 / 2+s}},
$$

where

$$
Z_{n, s}^{(3)}(u)=\sum_{k=0}^{s}\left(\begin{array}{c}
2 s+1 \\
s+k+1
\end{array}\right) u^{-(2 n+1) k} .
$$

The following result was conjectured in [11:

Theorem 2.4. For each fixed $\rho>1$ and $s \in \mathbb{N}_{0}$ there exists $n_{0}=n_{0}(\rho, s)$ such that

$$
\max _{z \in \mathcal{E}_{\rho}}\left|K_{n, s}^{(3)}(z)\right|=K_{n, s}^{(3)}\left(\frac{1}{2}\left(\rho+\rho^{-1}\right)\right)
$$

for each $n \geq n_{0}$. 
TABLE 3. The smallest possible (s.p.) values of $n_{0}$ and their approximations $\bar{t}(t$ is the largest zero of $G$ )

\begin{tabular}{|c|c|c|c|c|c|c|c|c|c|c|c|}
\hline \multirow[b]{2}{*}{$\rho$} & \multicolumn{10}{|c|}{ the s.p. $n_{0}$} & \multirow[b]{2}{*}{$\bar{t}$} \\
\hline & $s=1$ & $s=2$ & $s=3$ & $s=4$ & $s=5$ & $s=6$ & $s=7$ & $s=8$ & $s=9$ & $s=10$ & \\
\hline 1.01 & 726 & 726 & 728 & 728 & 728 & 730 & 730 & 730 & 730 & 730 & 732 \\
\hline 1.02 & 324 & 324 & 324 & 326 & 326 & 326 & 326 & 326 & 326 & 326 & 328 \\
\hline 1.03 & 200 & 202 & 202 & 202 & 202 & 202 & 202 & 202 & 202 & 202 & 204 \\
\hline 1.04 & 142 & 142 & 144 & 144 & 144 & 144 & 144 & 144 & 144 & 144 & 144 \\
\hline 1.05 & 110 & 110 & 110 & 110 & 110 & 110 & 110 & 110 & 110 & 110 & 110 \\
\hline 1.06 & 88 & 88 & 88 & 88 & 88 & 88 & 88 & 88 & 88 & 88 & 88 \\
\hline 1.07 & 72 & 72 & 74 & 74 & 74 & 74 & 74 & 74 & 74 & 74 & 74 \\
\hline 1.08 & 62 & 62 & 62 & 62 & 62 & 62 & 62 & 62 & 62 & 62 & 62 \\
\hline 1.09 & 54 & 54 & 54 & 54 & 54 & 54 & 54 & 54 & 54 & 54 & 54 \\
\hline 1.1 & 48 & 48 & 48 & 48 & 48 & 48 & 48 & 48 & 48 & 48 & 48 \\
\hline 1.2 & 20 & 20 & 20 & 20 & 20 & 20 & 20 & 20 & 20 & 20 & 20 \\
\hline 1.3 & 12 & 12 & 12 & 12 & 12 & 12 & 12 & 12 & 12 & 12 & 12 \\
\hline 1.4 & 8 & 8 & 8 & 8 & 8 & 8 & 8 & 8 & 8 & 8 & 8 \\
\hline 1.5 & 6 & 6 & 6 & 6 & 6 & 6 & 6 & 6 & 6 & 6 & 6 \\
\hline 1.6 & 4 & 4 & 6 & 6 & 6 & 6 & 6 & 6 & 6 & 6 & 6 \\
\hline 1.7 & 4 & 4 & 4 & 4 & 4 & 4 & 4 & 4 & 4 & 4 & 4 \\
\hline 1.8 & 4 & 4 & 4 & 4 & 4 & 4 & 4 & 4 & 4 & 4 & 4 \\
\hline 1.9 & 2 & 2 & 2 & 2 & 4 & 4 & 4 & 4 & 4 & 4 & 4 \\
\hline 2. & 2 & 2 & 2 & 2 & 2 & 2 & 2 & 2 & 2 & 2 & 2 \\
\hline 2.5 & 2 & 2 & 2 & 2 & 2 & 2 & 2 & 2 & 2 & 2 & 2 \\
\hline
\end{tabular}

Proof. Because of (2.15), it is sufficient to prove

$$
\frac{\left(a_{1}+\cos \theta\right)^{s+1}\left|Z_{n, s}^{(3)}\left(\rho e^{i \theta}\right)\right|}{\left(a_{2}-\cos 2 \theta\right)^{1 / 2}\left(a_{2 n+1}+\cos (2 n+1) \theta\right)^{1 / 2+s}} \leq \frac{\left(a_{1}+1\right)^{s+1} Z_{n, s}^{(3)}(\rho)}{\left(a_{2}-1\right)^{1 / 2}\left(a_{2 n+1}+1\right)^{1 / 2+s}}
$$

for sufficiently large $n\left(n \geq n_{0}(\rho, s)\right)$ and $\theta \in(0, \pi]$, where $a_{j}$ are given by (2.2).

It is obvious that for each $n \geq 1$, we have $\left(a_{1}+\cos \theta\right)^{s+1} \leq\left(a_{1}+1\right)^{s+1}$. On the basis of the results from Subsection 2.1, we obtain

$$
\begin{aligned}
& \frac{\left|Z_{n, s}^{(3)}\left(\rho e^{i \theta}\right)\right|}{\left(a_{2}-\cos 2 \theta\right)^{1 / 2}\left(a_{2 n+1}+\cos (2 n+1) \theta\right)^{1 / 2+s}} \\
& =\frac{\left|Z_{n+1 / 2, s}^{(1)}\left(\rho e^{i \theta}\right)\right|}{\left(a_{2}-\cos 2 \theta\right)^{1 / 2}\left(a_{2(n+1 / 2)}+\cos (2(n+1 / 2)) \theta\right)^{1 / 2+s}} \\
& \leq \frac{Z_{n+1 / 2, s}^{(1)}(\rho)}{\left(a_{2}-1\right)^{1 / 2}\left(a_{2(n+1 / 2)}+1\right)^{1 / 2+s}}=\frac{Z_{n, s}^{(3)}(\rho)}{\left(a_{2}-1\right)^{1 / 2}\left(a_{2 n+1}+1\right)^{1 / 2+s}},
\end{aligned}
$$

for each $n \geq n_{0}\left(n_{0}=n_{0}(\rho, s)\right)$. Therefore, we conclude that

$$
\left|K_{n, s}^{(3)}\left(\frac{1}{2}\left(\rho e^{i \theta}+\rho^{-1} e^{-i \theta}\right)\right)\right| \leq K_{n, s}^{(3)}\left(\frac{1}{2}\left(\rho+\rho^{-1}\right)\right)
$$

for each $n \geq n_{0}\left(n_{0}=n_{0}(\rho, s)\right)$.

If $t$ is the largest zero of $F$, for $n_{0}$ we can take $[(2 t-1) / 2]+1$. 


\section{ACKNOWLEDGMENT}

The authors would like to thank the referee for a careful reading of the manuscript and for his valuable comments.

\section{REFERENCES}

1. S. Bernstein, Sur les polynomes orthogonaux relatifs à un segment fini, J. Math. Pures Appl. 9 (1930), 127-177.

2. J. D. Donaldson and D. Elliott, A unified approach to quadrature rules with asymptotic estimates of their remainders, SIAM J. Numer. Anal. 9 (1972), 573-602. MR0317522 (47:6069)

3. W. Gautschi and G. V. Milovanović, S-orthogonality and construction of Gauss-Turán type quadrature formulae, J. Comput. Appl. Math. 86 (1997), 205-218. MR.1491435 (99a:65030)

4. W. Gautschi and S. E. Notaris, Gauss-Kronrod quadrature formulae for weight function of Bernstein-Szegö type, J. Comput. Appl. Math. 25 (1989), 199-224. MR988057 (90d:65045)

5. W. Gautschi and R. S. Varga, Error bounds for Gaussian quadrature of analytic functions, SIAM J. Numer. Anal. 20 (1983), 1170-1186. MR723834 (85j:65010)

6. W. Gautschi, E. Tychopoulos and R. S. Varga, A note on the contour integral representation of the remainder term for a Gauss-Chebyshev quadrature rule, SIAM J. Numer. Anal. 27 (1990), 219-224. MR1034931 (91d:65044)

7. A. Ghizzetti and A. Ossicini, Quadrature formulae, Akademie - Verlag, Berlin, 1970. MR 0269116 (42:4012)

8. D. B. Hunter, G. Nikolov, On the error term of symmetric Gauss-Lobatto quadrature formulae for analytic functions, Math. Comp. 69 (2000), 269-282. MR1642754 (2001a:65030)

9. G. V. Milovanović, Quadratures with multiple nodes, power orthogonality, and momentpreserving spline approximation, in Numerical Analysis in 20th Century, Vol. 5 (W. Gautschi, F. Marcellán, and L. Reichel, eds.), J. Comput. Appl. Math. 127 (2001), 267-286. MR1808578 (2002e:65039)

10. G. V. Milovanović and M. M. Spalević, Quadrature formulae connected to $\sigma$-orthogonal polynomials, J. Comput. Appl. Math. 140 (2002), 619-637. MR1934463 (2003h:65025)

11. G. V. Milovanović and M. M. Spalević, Error bounds for Gauss-Turán quadrature formulae of analytic functions, Math. Comp. 72 (2003), 1855-1872. MR1986808 (2004c:41068)

12. G. V. Milovanović, M. M. Spalević, An error expansion for Gauss-Turán quadratures and $L^{1}$-estimates of the remainder term, BIT 45 (2005), 117-136. MR2164228 (2006e:41058)

13. G. V. Milovanović, M. M. Spalević and A. S. Cvetković, Calculation of Gaussian quadratures with multiple nodes, Math. Comput. Modelling 39 (2004), 325-347. MR2037399 (2005g:65041)

14. A. Ossicini and F. Rosati, Funzioni caratteristiche nelle formule di quadratura gaussiane con nodi multipli, Boll. Un. Mat. Ital. (4) 11 (1975), 224-237. MR0408212 (53:11977)

15. T. Schira, The remainder term for analytic functions of Gauss-Lobatto quadratures, J. Comput. Appl. Math. 76 (1996), 171-193. MR1423516 (97m:41033)

16. T. Schira, The remainder term for analytic functions of symmetric Gaussian quadratures, Math. Comp. 66 (1997), 297-310. MR1372009 (97c:65050)

Department of Mathematics, University of Niš, Faculty of Electronic Engineering, P.O. Box 73, 18000 Niš, SERBiA

E-mail address: grade@elfak.ni.ac.yu

Department of Mathematics and Informatics, University of Kragujevac, Faculty of Science, P.O. Box 60, 34000 Kragujevac, Serbia

E-mail address: spale@kg.ac.yu

Department of Mathematics and Informatics, University of Banja Luka, Faculty of Science, M. Stojanovića 2, 51000 Banja Luka, Bosnia and Herzegovina

E-mail address: pranic77m@yahoo.com 